\title{
Generation of solitary waves by forward- and backward-step bottom forcing
}

\author{
By DAO-HUA ZHANG AND ALLEN T. CHWANG \\ Department of Mechanical Engineering, The University of Hong Kong, \\ Pokfulam Road, Hong Kong
}

(Received 1 February 2000 and in revised form 18 September 2000)

A finite difference method based on the Euler equations is developed for computing waves and wave resistance due to different bottom topographies moving steadily at the critical velocity in shallow water. A two-dimensional symmetric and slowly varying bottom topography, as a forcing for wave generation, can be viewed as a combination of fore and aft parts. For a positive topography (a bump), the fore part is a forward-step forcing, which contributes to the generation of upstream-advancing solitary waves, whereas the aft part is a backward-step forcing to which a depressed water surface region and a trailing wavetrain are attributed. These two wave systems respectively radiate upstream and downstream without mutual interaction.

For a negative topography (a hollow), the fore part is a backward step and the aft part is a forward step. The downstream-radiating waves generated by the backwardstep forcing at the fore part will interact with the upstream-running waves generated by the forward-step forcing at the aft. Therefore, the wave system generated by a negative topography is quite different from that by a positive topography. The generation period of solitary waves is slightly longer and the instantaneous drag fluctuation is skewed for a negative topography. When the length of the negative topography increases, the oscillation of the wave-resistance coefficient with time does not coincide with the period of solitary wave emission.

\section{Introduction}

The study of solitary waves generated by moving objects or bottom topographies has received much attention since the initial experiments of Huang et al. (1982) and the remarkable numerical findings of Wu \& Wu (1982). Wu \& Wu (1982) studied forced waves in a transcritical state using the generalized Boussinesq $(\mathrm{g}-\mathrm{B})$ model proposed by $\mathrm{Wu}$ (1981). They identified numerically the existence of a train of solitions of equal size moving ahead of a bump. Immediately behind the bump there is a flat depression zone followed by a train of cnoidal-like waves. The other widely used theoretical model for studying the phenomenon of upstream-running solitary waves is the forced Korteweg-de Vries (fKdV) equation, which is simpler than the g-B model with the further assumption of waves propagating in one direction only. Because of its simplicity, this model has been used to study various types of forcing by Akylas (1984), Cole (1985), Grimshaw \& Smyth (1986) among others and in a more general form by $\mathrm{Wu}$ (1987). The theoretical models admit as external forcing disturbances the surface pressure and topography, which are assumed to be positive or negative functions. Lee, Yates \& Wu (1989) made an extensive quantitative comparison between numerical results of the fKdV and g-B models and experiments on transcritical upstream soliton radiation. Their comparison showed that the agreement between the theoretical 
models, when the viscous effect was crudely accounted for, and the experiments was generally very good. They found that for the fKdV model, the surface pressure and bottom topography were entirely equivalent, whereas for the g-B model the surface pressure acted as a stronger disturbance than the bottom bump of the same distribution, thereby producing larger waves in a shorter period.

The experimental study of Ertekin, Webster \& Wehausen (1984) of a ship in a channel indicated that the blockage coefficient (defined as the ratio of the mid-shipsectional area to the cross-sectional area of the water channel) was the dominant forcing factor in governing soliton generation, but not the sole one as the hull displacement also played an important role. This was shown in figures 9 and 11 of Ertekin et al. (1984): the blockage coefficients for plots $(a)$ and $(b)$ in these figures were the same, but the displacements were different due to different draughts. Therefore, the difference between the plots solely indicated the effect of displacement on the wave generation.

Grimshaw \& Smyth (1986) conducted asymptotic studies on forced flows in a stratified fluid. They showed that the forcing function of the fKdV model was characterized by two parameters: forcing amplitude $G_{0}$ (in a single-layer flow, when scaled by water depth, $G_{0}$ stands for the blockage coefficient) and a positive parameter $\xi$ that measures the lengthscale of the forcing. They found, based on the mass and energy conservation argument, approximate formulas for the depth of the downstream depression and the amplitude of the upstream solitons as functions of the forcing velocity, the length and amplitude of the forcing. The dependence of the wave mechanical energy and drag on the area and speed of the forcing was explicitly calculated by Shen (1996). Smyth (1987) modelled the upstream flow by a modulated cnoidal wavetrain, not by a series of equal KdV solitary waves as in Grimshaw \& Smyth (1986), and obtained improved solutions for the upstream flow. He found similar approximate formulas for the amplitude and period of soliton generation as Grimshaw \& Smyth (1986). Teng \& Wu (1997) studied the effect of forcing length on the wave amplitude and period of generation based on the Boussinesq and KdV models. They showed that when the length is much greater than the water depth, it has little effect on the wave amplitude and period. If the length is of the same order as the water depth, the effect of disturbance length becomes important. Shen (1992) also indicated that the length of disturbance might have an important effect on solitary wave generation.

While the surface pressure and the bottom bump show some difference in the strength of disturbance in different theoretical models, the features of solitary waves so generated are very similar. However, the features of wave systems generated by positive or negative forcing functions are quite different. Grimshaw \& Smyth (1986), Wu (1987), and Camassa \& Wu (1991) studied solitary waves generated by a negative forcing function and gave descriptions of their features.

A numerical method which solves the Navier-Stokes equations (or Euler equations) by direct integration with consistent free-surface boundary conditions for time-dependent viscous (or inviscid) free-surface flows was developed by Zhang \& Chwang $(1996,1999)$. The method was validated by the experimental data of Lee et al. (1989). The computational results from the direct numerical integration of the Navier-Stokes equations were compared with the experimental data and the numerical results of the fKdV and g-B models of Lee et al. (1989) at the transcritical range. Since viscous effects are accurately modelled in the Navier-Stokes equations, the agreement of the numerical results by Zhang \& Chwang (1996) with the experimental data was excellent and much better than the numerical results of the fKdV and g-B models. The method was employed to study the general characteristics of soli- 
tary waves forced by underwater moving objects including viscous effects. The phase velocity and the period of solitary wave generation also showed good agreement with theoretical predictions (Zhang \& Chwang 1999).

In the present paper, the basic mechanism underlying the features of solitary waves generated by bottom topographies is explored by the numerical model of Zhang \& Chwang (1999). The geometry of the topographies (analogous to the forcing function in theoretical models) is simply part of the boundaries of the calculation domain, which can be easily and accurately simulated in the model. The investigation has three aims: $(a)$ to describe the dependence of the upstream-advancing solitons on the height and length of the topography, $(b)$ to explore whether the waves generated by a bump or a hollow forcing are a superposition of the waves due to two step forcings, and $(c)$ to explain, with the new findings in $(b)$, why the wave systems due to a positive forcing are different from those due to a negative forcing.

Descriptions of the computational method are provided briefly in $\S 2$. Section 3 describes and discusses the results on waves generated by various bottom topographies including positive, negative, forward-step and backward-step forcings. Quantitative comparisons between numerical solutions for the amplitude of the lead upstream wave for various forcing heights and the approximate solutions of Smyth (1987) and Grimshaw \& Smyth (1986) are made in $\S 3.1$. Section 3.2 presents the new finding that the waves generated by a bump or a hollow forcing are a superposition of the waves due to forward- and backward-step forcings formed by cutting the bump or hollow in half at the middle and joining the cut ends to a horizontal portion of the bottom (figure 1). The dependence of the wave amplitude on the forcing length is also discussed. In $\S 3.3$, the difference between wave systems due to positive and negative forcings is explained. Finally, conclusions are given in $\S 4$.

\section{Outline of the computational method}

The model of Zhang \& Chwang (1999) with the Euler formulation is employed in the present study. The following provides a summary of the method. For more detailed information refer to Zhang \& Chwang $(1996,1999)$.

The unsteady Euler and continuity equations are solved with nonlinear kinematic and dynamic free-surface boundary conditions in a body/free-surface fitted grid. The grid is allowed to move with the free surface, providing a more accurate treatment of the free-surface boundary conditions. The Cartesian coordinates $(x, y)$ of the physical domain are transformed to the numerically generated, boundary-fitted, non-orthogonal curvilinear coordinates $(\xi, \eta)$ in the computational domain, while the velocity components $(u, v)$ in the Cartesian coordinate system of the physical domain remain unchanged. The transformed equations, in a conservation form in the boundary-fitted coordinate system, are solved using a regular grid. The second-order central difference is used to discretize the spatial differentials while the convection terms are discretized by the QUICK scheme. Velocity components are updated explicitly by the time-splitting fractional-step method, while pressure is updated by solving a Poisson equation for the pressure increment between two adjacent time steps. The grid is updated at each time step to conform to both the body and the free surface.

\section{Results and discussion}

A two-dimensional symmetric and slowly varying bottom topography, as a forcing for wave generation, can be looked upon as a combination of fore and aft parts. For 
(a)

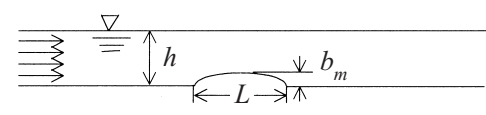

(b)

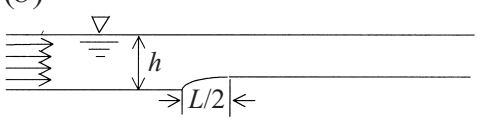

(c) (d)

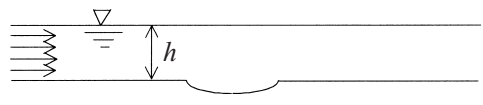

(e)

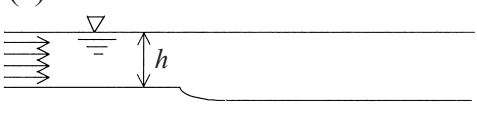

$(f)$

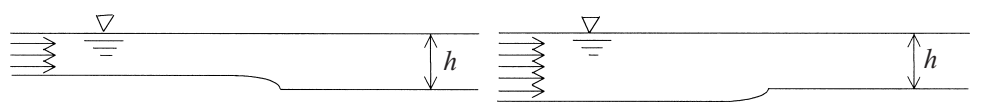

FIGURE 1. A sketch of bottom topographies: $(a)$ positive; $(d)$ negative; $(b, f)$ forward step; $(c, e)$ backward step.

a positive topography (a bump), the fore part is a forward step and the aft part is a backward step. On the other hand, for a negative topography (a hollow), the fore part is a backward step and the aft part is a forward step. A sketch of different bottom topographies is shown in figure 1 .

Bottom topographies used for the present numerical computations are either

$$
b(x)= \pm \frac{b_{m}}{2}\left[1+\cos \left(\frac{2 \pi}{L} x\right)\right] \text { for } \quad-L / 2 \leqslant x \leqslant L / 2
$$

or

$$
b(x)=b_{m} \operatorname{sech}^{2} x \text { for }-\infty<x<\infty .
$$

The undisturbed water depth $h$ is normalized to $1, b_{m}$ is the maximum height of the disturbance scaled by water depth, which is the blockage coefficient in the twodimensional case, and $L$ is the disturbance length scaled by water depth. Only the exact linear resonant case is considered in the present study. Thus, the Froude number defined as $F=U_{0} / \sqrt{g h}$ is equal to 1 for all calculations, where $U_{0}$ is the upstream uniform inflow velocity and $g$ the gravitational constant. The calculation domain is of one unit length in the vertical direction and 180 unit lengths in the horizontal direction. Forty-one grid points are used in the vertical direction and 397 grid points in the horizontal direction. Time is scaled by $h / U_{0}$ and the time step is set to be $\Delta T=0.01$.

\subsection{Positive forcing}

Typical numerical results for the topography given by equation (2) with $b_{m}=0.1$ are shown in figure 2(a). If the height of the topography is assumed to be zero when it is less than 0.0004 , then the length of the topography $L$ is approximately equal to 6.8. This is equivalent to setting $\xi=0.3$ (the half-width of the forcing $\xi^{-1} \approx 3.3$ ) in the study of Grimshaw \& Smyth (1986). The general features of the upstream periodically radiating solitary waves forced by a steadily moving two-dimensional disturbance sustained at resonance are clearly shown.

The theoretical and numerical study of Grimshaw \& Smyth (1986) showed that the forcing function of the $\mathrm{fKdV}$ model is characterized by two parameters: amplitude $G_{0}$ and a positive parameter $\xi$ ( $\xi$ measures the length scale of the forcing, i.e. $\xi^{-1}$ is 

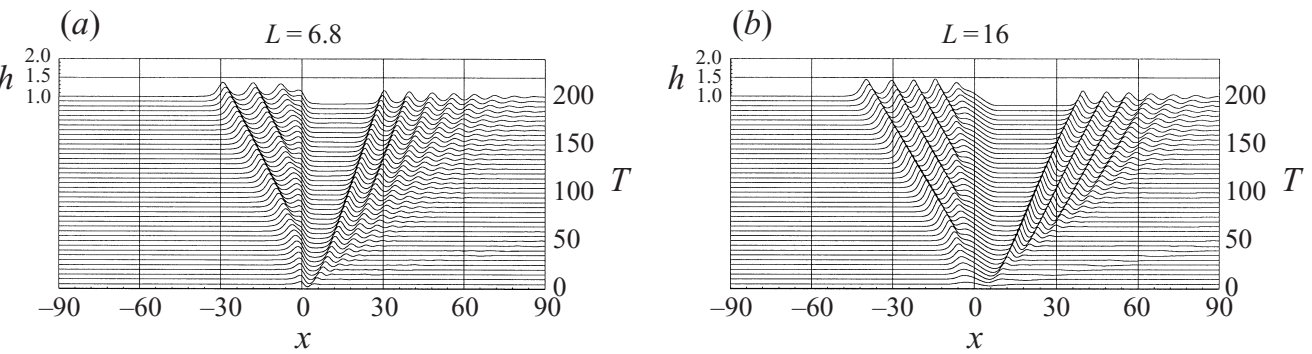

FIGURE 2. Wave profiles generated by ( $a$ ) a sech ${ }^{2}$-shaped bump with height $b_{m}=0.1$ and length $L=6.8$ and $(b)$ a cosine-shaped bump with height $b_{m}=0.1$ and length $L=16$.

its half-width). These two parameters determine the area of the forcing distribution integrated over the base line, which represents the intensity of the forcing. When scaled by water depth, the amplitude of the forcing function $G_{0}$ stands for the blockage coefficient. For a fixed value of $G_{0}$, the wider the forcing, the larger the area of the forcing distribution and the stronger the forcing intensity will be. When the detuning parameter $\Delta=0$ (at exact linear resonance) Grimshaw \& Smyth (1986) found from their mass and energy conservation argument that for a broad obstacle $(\xi \rightarrow 0)$, the amplitude of the upstream-advancing solitary waves was determined solely by the blockage coefficient, i.e.

$$
a \approx 1.15 \sqrt{G_{0}} .
$$

For a narrow obstacle $(\xi \rightarrow \infty)$, the amplitude was determined by the dimensionless area of the forcing distribution, i.e.

$$
a \approx\left(G_{0} K \xi^{-1}\right)^{2 / 3}
$$

where $K$ is the integral of the forcing function from minus infinity to plus infinity. They compared the solitary-wave amplitude $a$ obtained from the approximate expression (3) to their numerical results obtained for forcing function $\operatorname{sech}^{2}(\xi X)$ with $\Delta=0$, $\xi=0.3$, and $G_{0}=0.5,1.0,1.5$ and 2.0 respectively. The agreement was good.

For $\xi \ll 1$, Grimshaw \& Smyth (1986) found that the numerical solutions of the fKdV equation with either $\operatorname{sech}^{2}(\xi X)$ or $\exp (-\xi X)^{2}$ forcing functions were quantitatively in agreement. Shen $(1991,1996)$ approximated a short bump by $P \delta(x)$ in the fKdV equation, where $P$ is the dimensionless area of the bump and $\delta(x)$ is the Dirac delta function. He showed that, for the locally forced case, the surface wave was independent of the shape of the bottom obstruction, but instead depended on its area.

Smyth (1987) modelled the upstream flow by a modulated cnoidal wavetrain and obtained an improved solution for the upstream flow. For a broad forcing (small $\xi$ ) with $\Delta=0$, he found that the amplitude of solitons at the head of the expansion fan was given as

$$
a \approx 1.25 \sqrt{G_{0}} .
$$

To compare the present numerical results for the upstream flow with those given by approximate expressions (3) and (5), calculations with the topography given by equation (2) and four forcing amplitudes, i.e. $b_{m}=0.05,0.10,0.15$ and 0.20 , are carried out. The amplitudes of the leading upstream waves are presented in table 1 together 


$\begin{array}{cccc}b_{m} & a, \text { numerical results } & a \approx 1.15 \sqrt{b_{m}} & a \approx 1.25 \sqrt{b_{m}} \\ 0.05 & 0.263 & 0.257 & 0.280 \\ 0.10 & 0.376 & 0.364 & 0.395 \\ 0.15 & 0.458 & 0.445 & 0.484 \\ 0.20 & 0.530 & 0.514 & 0.559\end{array}$

TABLE 1. A comparison between numerical results for the solitary-wave amplitude $a$ and those by the approximate expressions for a broad forcing. The numerical results were obtained for the forcing function given by equation (2) with $F=1.0$.

with the values obtained from approximate expressions (3) and (5). The agreement is good. The present numerical results are between the two approximate expressions.

\subsection{Forward- and backward-step forcings}

Shen (1992) indicated that the length of the disturbance might have an important effect on the solitary wave generation. The numerical results of Teng \& Wu (1997) based on the Boussinesq and $\mathrm{KdV}$ models showed that when the length is sufficiently large (i.e. much greater than the water depth), it has little effect on the wave amplitude and period, and the dominant forcing factor is indeed the blockage coefficient. However, if the length is relatively short (i.e. same order as the water depth), the blockage coefficient is no longer the sole dominant forcing factor as the effect of the disturbance length also becomes important: the shorter the disturbance length, the weaker the forcing.

In the present study, flows over positive cosine-shaped topographies given by equation (1) with height $b_{m}=0.1$ and length $L=2,4,8,12$, and 16 respectively are calculated. It is found that for $L$ less than 12, the amplitude of the upstreamadvancing solitary waves increases, but the period of wave generation decreases, as $L$ increases. When $L$ is equal to or greater than 12 , the waves generated by the topography are almost the same: they are independent of the disturbance length. Here, only the results for $L=16$ are presented in figure $2(b)$.

The wave systems shown in figure 2 are generated by two different positive topographies with the same forcing amplitude but different forcing length. Although the number of upstream-advancing solitary waves, their amplitude and period of generation are different, the general features of the whole wave system are the same: it consists of upstream-advancing solitary waves and a depressed water region of nearly uniform depth, followed by a train of cnoidal-like waves downstream. The upstream and downstream radiating waves do not interact each other, indicating that the fore and aft parts of the topography contribute separately to upstream and downstream radiating waves.

Consider a positive topography (figure $1 a$ ) that cut at its maximum height at the middle and then joined to a horizontal bottom to form a forward step (figure $1 b$ ) and a backward step (figure 1c). In figure 3 , waves generated by these cosine-shaped forward- and backward-step forcings with $b_{m}=0.1$ and $L / 2=1.0$ are presented. It is interesting to see that the forward-step forcing only generates solitary waves with practically no depressed water region and trailing wavetrain. The excess mass for each soliton is $4(a / 3)^{1 / 2}$, where $a$ is the solitary wave amplitude. In the present case, $a \approx 0.5$, and 5.5 solitons were generated within $T=200$. The total excess mass of the 5.5 solitons is $5.5 \times 4(0.5 / 3)^{1 / 2}=9$. The area swept by the disturbance is $b_{m} F T=0.1 \times 1.0 \times 200=20$, and so the amount of mass that flows out downstream 

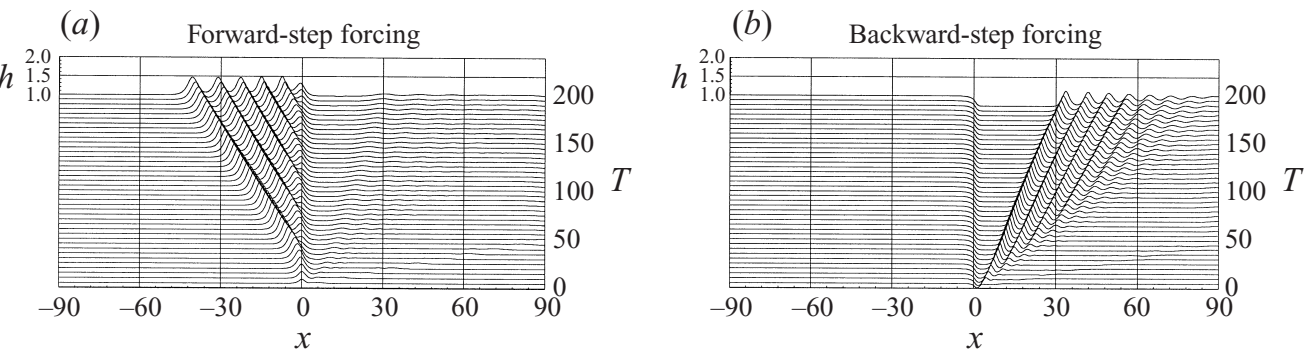

FIGURE 3. Wave profiles generated by $(a)$ forward-step- and $(b)$ backward-step-topographies with height $b_{m}=0.1$ and the length of the cosine portion $L / 2=1.0$.

is then 11. If it is assumed that the water surface for $x>0$ is flat and the water depth is $h$, the outflow velocity at the downstream boundary must be larger than the inflow velocity $F=1.0$. By mass conservation, the excess velocity $\delta u$ at the downstream boundary satisfies $\delta u T\left(h-b_{m}\right)=11$. This gives $\delta u=0.06$. The total outflow velocity at the downstream boundary obtained from the numerical calculation is 1.05 . This value seems to be rational from the rough analysis of mass conservation.

In contrast, the backward-step forcing only generates the depressed water region and the trailing wavetrain without the upstream-advancing solitary waves. Since the lengths of the forward- or backward-step forcing disturbance are semi-infinite, the combined wave system is similar to that generated by a long forcing as shown in figure $2(b)$. This indicates that the wave systems generated by a localized long forcing disturbance can be thought of as a combination of wave systems generated by a forward- and a backward-step forcing disturbance. This finding is important in understanding the role of different parts of the forcing in wave generation, and it will help to explain why the characteristics of wave systems generated by a negative forcing are different from those by a positive forcing. This will be discussed in the following section.

\subsection{Negative forcing}

Theoretical studies have shown that features of wave systems generated by a negative forcing function are quite different from those of a positive forcing. The following detailed and vivid description of the features of negative compared to positive forcing was given by $\mathrm{Wu}$ (1987): (a) the generation period of solitary waves is slightly longer; (b) the instantaneous drag fluctuation is skewed in phase (suggesting that it has two or more harmonics); $(c)$ the depressed water region behind the forcing is not as uniform in depth, owing to a succession of very small waves traversing backward across the region, reflected from the forcing; and $(d)$ the local wave continues to be excited to a relatively quite large amplitude within the region of the (negative) forcing before it breaks away, and then settles to a smaller height, as if overcoming a threshold.

The above description summarizes the overall features of wave systems generated by a negative forcing from theoretical models. In the present study, flow over a negative cosine-shaped topography given by equation (1) with length $L=2$ is calculated and the wave system so generated is shown in figure 4 together with the wave-resistance coefficient of the topography. It is seen that the features of the wave systems are different from those of a positive forcing and are in accord with the description by Wu (1987). A negative forcing (figure 1d) can be looked upon as a concave backwardstep forcing (figure 1e) followed by a concave forward-step forcing (figure $1 f$ ). In contrast to a positive forcing, the forward-step forms the aft part of the forcing 
(a)

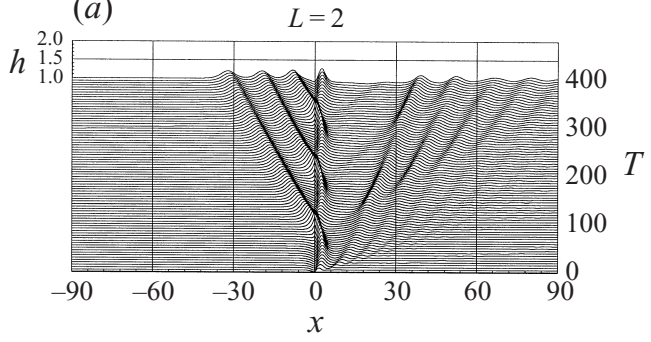

(b)

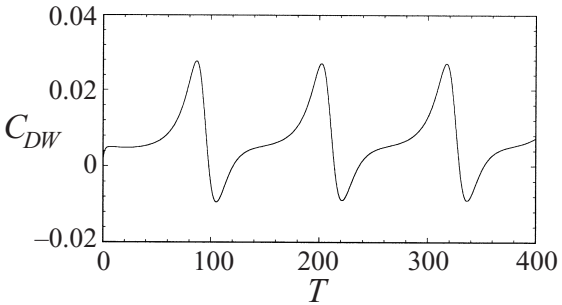

FiguRE 4. (a) Wave profiles and (b) wave resistance coefficient $C_{D W}$ due to a negative cosine-shaped topography with height $b_{m}=-0.1$ and length $L=2$.

disturbance while the backward-step forms the fore part. As discussed in the previous section, a forward-step forcing contributes to the generation of upstream-advancing solitary waves. In the case of a negative forcing, waves start to be generated just behind the aft part, first propagating downstream a small distance and then upstream across the negative forcing region. In the meantime, waves are generated at the fore part, radiating downstream and merging with the upstream-advancing waves. The wave continues to grow to relatively large amplitude within the region of the negative forcing as the energy acquired by local fluid at the rate of work done by moving disturbance accumulates. When the wave reaches a certain threshold amplitude, the increase in phase velocity with increasing amplitude will be sufficient to make the wave radiate out and finally settle to an upstream-advancing solitary wave of smaller height. However, in the case of a positive forcing, waves generated at the fore and aft parts radiate upstream and downstream respectively without mutual interaction. The difference in the process of wave generation and propagation between positive and negative forcings makes the generation period of solitary waves slightly longer in the case of a negative forcing.

Wave resistance of the forcing disturbance varies periodically with time, and the variation is related to the relative positions of the wave and the forcing. When a wave starts to be generated behind the aft part of the forcing, the corresponding wave resistance is small and positive. As the wave moves upstream, the wave resistance increases slowly at first, and then rapidly reaches a maximum when the highest elevation of the free surface is at the trailing edge of the forcing disturbance. This makes the resistance curve skew. As the wave moves further upstream away from the trailing edge, the wave resistance decreases rapidly and reaches zero when the wave is at the middle of the forcing region. It continues to drop to a negative maximum when the wave is at the leading edge of the forcing, and then starts to increase again as the wave breaks away from the forcing. This is followed by another solitary wave going through the same cycle. Although the instantaneous wave drag fluctuation is skewed, the periodic nature of the solitary wave generation is clearly locked to the evolution of the wave resistance coefficient.

For positive forcings, when $L$ is not sufficiently large, an increase or decrease of $L$ would strengthen or weaken the intensity of forcing, resulting in a change of the wave amplitude and period of wave generation, but no change in the features of wave generation. This does not seem to be true for a negative forcing. Figure 5 shows the wave system generated by a negative cosine-shaped distribution with length $L=4$ together with the wave-resistance coefficient of the forcing. It is seen from figure 5 that the features of the wave system are somewhat different from those 


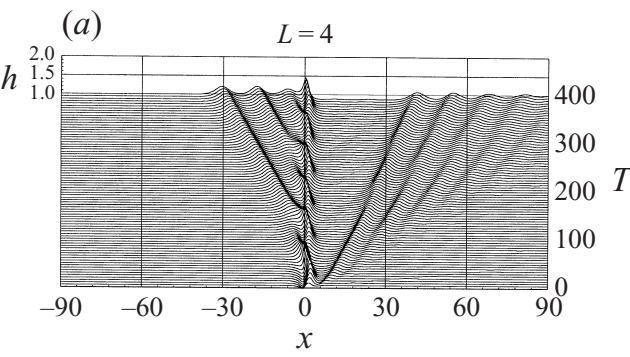

(b)

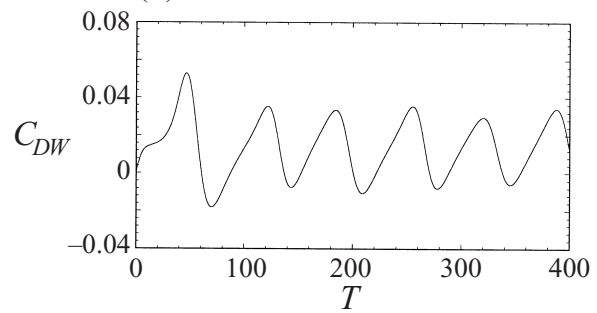

Figure 5. As figure 4 but for length $L=4$.

with length $L=2$ shown in figure 4 . When $L$ becomes larger, the nonlinear effect has a significant influence on the incipient emission time. The first wave generated at the aft part of the forcing disturbance dies out. The second wave probably gains mass and energy from the dying first wave as it propagates upstream. After this incipient emission, the regular sequential emission will then be ensured with every second incubating wave in the vicinity of the aft part of the forcing emitting a solitary wave. In this case, the oscillation of the wave-resistance coefficient with time does not coincide with the period of solitary wave emission. The emission occurs at every second peak of the wave-resistance curve. These salient features of the process were first explored by Camassa \& Wu (1991) based on numerical solutions of the fKdV model. Adopting the fKdV model and using two of its steady forced solitary wave solutions as primary flows, the stability of these two transcritical steady motions was investigated. It was found that if the rest state was chosen as the initial condition, the period of oscillation in the wave-resistance coefficient coincides with the period of birth of upstream-running solitary waves, whereas the incipient emission of an upstream-advancing solitary wave was delayed with a non-zero initial condition due to the nonlinear effect. The smaller the initial departure from the stationary response, the longer the local fluctuation would last before the first solitary wave became mature and was emitted.

\section{Conclusions}

The features of water waves generated by different bottom topographies moving steadily with the critical velocity through a layer of shallow water are studied numerically with the Euler equations. It is found that a forward-step forcing only generates upstream-running solitary waves and a backward-step forcing only generates a downstream-radiating depressed water region followed by a cnoidal wavetrain. Thus, a positive forcing disturbance-a combination of a forward-step forcing as the fore part and a backward-forcing as the aft part-would generate wave systems at its fore and aft parts respectively radiating out in opposite directions (upstream and downstream) separately without interaction. In contrast, a negative forcing disturbance-a combination of a concave backward-step forcing as the fore part and a concave forward-step forcing as the aft part-would generate upstream-running waves from its aft part (forward-step forcing) and downstream-radiating waves from its fore part (backward-step forcing). These two wave systems would interact in the negative forcing region causing the resultant waves to be quite different from those due to a positive forcing. 
This research was sponsored by the Hong Kong Research Grants Council under Grant Numbers HKU 568/96E, HKU 7066/97E and NSFC/HKU 8.

\section{REFERENCES}

AKYLAS, T. R. 1984 On the excitation of long nonlinear water waves by a moving pressure distribution. J. Fluid Mech. 141, 455-466.

Camassa, R. \& Wu, T. Y. 1991 Stability of forced steady solitary waves. Phil. Trans. R. Soc. Lond. A 337, 429-466.

Cole, S. L. 1985 Transient waves produced by flow past a bump. Wave Motion 7, 579-587.

Ertekin, R. C., Webster, W. C. \& Wehausen, J. V. 1984 Ship-generated solitons. Proc. 15th Symp. Naval Hydrodyn., Hamburg, pp. 347-364.

Ertekin, R. C., Webster, W. C. \& Wehausen, J. V. 1986 Waves caused by a moving disturbance in a shallow channel of finite width. J. Fluid Mech. 169, 275-292.

Grimshaw, R. H. J. \& Smyth, N. F. 1986 Resonant flow of a stratified fluid over topography. J. Fluid Mech. 169, 429-464.

Huang, D. B., Sibul, O. J., Webster, W. C., Wehausen, J. V., Wu, D. M. \& Wu, T. Y. 1982 Ships moving in the transcritical range. Proc. Conf. on Behavior of Ships in Restricted Waters, Vol. 2, pp. 26-1-26-10. Bulgarian Ship Hydrodynamic Center.

LEE, S. J., YATES, G. T. \& Wu, T. Y. 1989 Experiments and analyses of upstream-advancing solitary waves generated by moving disturbances. J. Fluid Mech. 199, 569-593.

MeI, C. C. 1986 Radiation of solitons by slender bodies advancing in a shallow channel. J. Fluid Mech. 162, 53-67.

SHEN, S. S. P. 1991 Locally forced critical surface waves in channels of arbitrary cross section. Z. Angew. Math. Phys. 42, 122-138.

SHEN, S. S. P. 1992 Forced solitary waves and hydraulic falls in two-layer flows. J. Fluid Mech. 234, 583-612.

SHEN, S. S. P. 1996 Energy distribution for waves in transcritical flows over a bump. Wave Motion 23, 39-48.

Smyth, N. F. 1987 Modulation theory solution for resonant flow over topography. Proc. R. Soc. Lond. A 409, 79-97.

Teng, M. H. \& WU, T. Y. 1997 Effect of disturbance length on resonantly forced nonlinear shallow water waves. Intl J. Offshore and Polar Engng 7, 262-268.

Wu, D. M. \& Wu, T. Y. 1982 Three-dimensional nonlinear long waves due to moving surface pressure. Proc. 14th Symp. on Naval Hydrodyn., pp. 103-125. National Academy Press.

WU, T. Y. 1981 Long waves in ocean and coastal waters. J. Engng Mech. Div. ASCE 107, 501-522.

WU, T. Y. 1987 Generation of upstream advancing solitons by moving disturbances. J. Fluid Mech. 184, 75-99.

ZhaNG, D. H. \& Chwang, A. T. 1996 Numerical study of nonlinear shallow water waves produced by a submerged moving disturbance in viscous flow. Phys Fluids 8, 147-155.

Zhang, D. H. \& Chwang, A. T. 1999 On solitary waves forced by underwater moving objects. J. Fluid Mech. 389, 119-136. 